\title{
A Timing-Driven Floorplanning Algorithm with the Elmore Delay Model for Building Block Layout
}

\author{
Tetsushi KOIDE, Shin'ichi WAKABAYASHI \\ Faculty of Engineering, Hiroshima University \\ 4-1, Kagamiyama 1 chome, Higashi-Hiroshima 739, JAPAN, \\ Phone:+81-824-24-7676, FAX:+81-824-22-7195, \\ e-mail:koide@ecs.hiroshima-u.ac.jp
}

\begin{abstract}
This paper presents a timing-driven floorplanning algorithm for building block layout. As the interconnection delay model, the proposed algorithm adopts the Elmore delay model. The algorithm consists of two phases. In phase 1, a timing-driven topological arrangement of blocks is generated with the resolution of overlap among blocks using nonlinear programming under the timing constraints. In phase 2, the algorithm performs floorplan sizing which determines the sizes and the shapes of blocks based on the topological arrangement obtained in phase 1 so as to minimize the chip area, and obtains a legal floorplan. This phase is based on the topological constraint manipulation. Through the experimental results, the proposed algorithm can produce results without any timing violations within a practical computation time.
\end{abstract}

\section{Keywords}

Floorplanning, Elmore delay, nonlinear programming, topological constraint

\section{INTRODUCTION}

In building block layout, a VLSI circuit is partitioned into a set of components, referred to as blocks, and there are two types of blocks, that is, hard blocks and soft blocks. For the former, they are pre-designed and the shapes and the pin positions of the blocks are pre-determined. For the latter, since they are not pre-designed, we need to specify the exact shapes and the pin positions in layout design. The goal of building block layout design is to determine the shapes of blocks, the positions of blocks, and the interconnections among blocks so that the chip area is utilized in the best possible way.

In the building block layout design, floorplanning is the first and an essential design step to determine several important factors of the layout such as the overall required area of a chip, the sizes and shapes of blocks, pin and 
pad locations, etc. The goal of floorplanning is to realize a placement plan that will decide topological proximity as well as appropriate shapes of blocks. Floorplanning is usually divided into two steps (Lengauer 1990), which are generally called global placement and detailed placement. In the global placement step, we determine the relative positions of blocks so as to minimize the total wire length. Since blocks are generally treated as points, which are the center coordinates of the blocks, and are placed in the chip, the blocks may be overlapped each other because of their variety of the shapes and the areas. On the other hand, the detailed placement essentially converts a global placement floorplan into a legal optimal area floorplan* which respects a placement produced in the global placement step.

Due to the progress of semi-conductor process technologies, interconnection delay cannot be ignored as well as switching delay of gates in the physical design(Bakoglu 1990). Thus we need to explicitly consider the interconnection delay during layout design. Similar to the placement step, the floorplanning step has a large effect on the performance of a circuit. Therefore several timingdriven algorithms have been proposed (Prasitjutrakul \& Kubitz 1989, Ogawa et al. 1990, Gao et al. 1992, Tia \& Liu 1993, Sait et al. 1994, Youssef et al. 1995).

In this paper, we propose a timing-driven floorplanning algorithm for the building block layout. The proposed algorithm adopts the Elmore delay model as the interconnection delay model. The proposed floorplanning algorithm consists of two phases: (1) construction of a timing-driven topological arrangement with nonlinear programming, (2) conversion of the topological arrangement into a legal floorplan. In the first phase, we determine a topological arrangement of blocks using nonlinear programming considering the timing constraints and the resolution of overlap among blocks. Next, we perform floorplan sizing which determines the sizes and the shapes of blocks based on the topological arrangement obtained in the first phase so as to minimize the chip area and obtain a legal floorplan. The second phase is based on the topological constraint manipulation. From the experimental results compared with a non-timing-driven floorplanning algorithm, the proposed floorplanning algorithm can produce floorplans without any timing violations in a practical computation time.

The remainder of this paper is organized as follows. In Section 2, we define the interconnection delay model and the timing constraint, and formulate the floorplanning problem. Section 3 presents a timing-driven floorplanning algorithm with nonlinear programming and topological constraint manipulation. Section 4 shows experimental results and the evaluation of the proposed algorithm. Finally, in Section 5, we conclude this paper and describe future work.

*We define a floorplan to be legal if there are no overlaps among the blocks and the shapes and placement of the blocks satisfy the aspect ratio bounds and pre-placement conditions. 


\section{PROBLEM FORMULATION}

A chip is a rectangular region which is surrounded by $\mathrm{I} / \mathrm{O}$ pins on its boundary and contains blocks of different sizes within this boundary. Let $\mathcal{L}=(\mathcal{M}, \mathcal{N})$ be a logic circuit, where $\mathcal{M}$ is a set of blocks and $\mathcal{N}$ is a set of nets. A set of blocks $\mathcal{M}=\left\{m_{1}, m_{2}, \cdots, m_{M}\right\}$ consists of a set of hard blocks $\mathcal{M} h$ and a set of soft blocks $\mathcal{M}$ s. Let $w\left(m_{j}\right)$ and $h\left(m_{j}\right)$ be the width and the height of block $m_{j}$, respectively, and let $a\left(m_{j}\right)$ and $r\left(m_{j}\right)$ be the area and the aspect ratio of block $m_{j}$, respectively. For hard block $m h_{i} \in \mathcal{M} h$, its width and height are fixed and the pin positions are specified in advance. For soft block $m s_{i} \in \mathcal{M} s$, its width and height are temporarily given but not fixed, and its area and lower and upper bounds of its aspect ratio, $\left(r_{l}\left(m s_{i}\right), r_{u}\left(m s_{i}\right)\right)$, are specified. A set of nets $\mathcal{N}=\left\{n_{1}, n_{2}, \cdots, n_{N}\right\}$ describes the connectivity information. For simplicity in the presentation, it is assumed that all the pins of a block are located at the center of the block. We assume that the interconnections are realized by using two layers, the first metal layer (M1) and the second metal layer (M2). The M1 layer is mainly used for horizontal wiring and the M2 layer is mainly for vertical wiring.

An equivalent circuit of an interconnection is originally modeled as a distributed RC circuit, and the Elmore's delay equation(Elmore 1948) is often used to represent the interconnection delay (Figure 1). When a multi-terminal net $n_{i}$ is implemented by a Steiner tree, Kuh et al. give an upper bound of the Elmore delay from the source pin to a load pin $j$ of net $n_{i}$ (Kuh \& Shih 1992).

We employ the equation given by Kuh et al. as the interconnection delay model. However, it is not practical to construct Steiner trees during floorplanning from the point of computation time. Therefore the wire length of net $n_{i}$ is estimated with half the perimeter length of a bounding box of enclosing the pins of net $n_{i}$; the path length from the source to a load $j$ of net $n_{i}$ with half the perimeter length of a bounding box enclosing the source pin and load pin $j$. Figure 1 shows an example of the estimation of the wire length. Then the delay from the source pin to load pin $j$ of net $n_{i}$ is defined as,

$\operatorname{del}_{i j}\left(w_{i}, h_{i}, l 1_{i j}, l 2_{i j}\right)=\left(c_{1} w_{i}+c_{2} h_{i}+\sum_{k} C_{l k}\right)\left(R_{i 0}+r_{1} l 1_{i j}+r_{2} l 2_{i j}\right)$
where

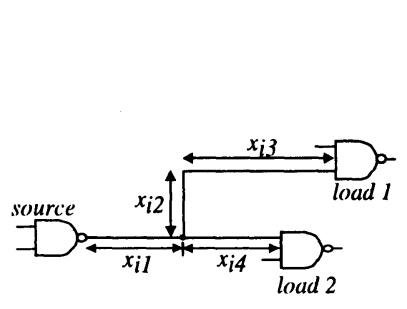

(a)

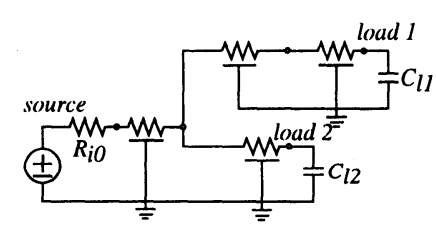

(b)

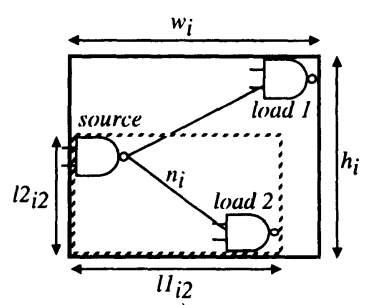

(c)

Figure 1 Interconnection delay model. 
$w_{i}, h_{i} \quad$ : the width and height of the bounding box of net $n_{i}$, respectively. $l 1_{i j}, l 2_{i j}$ : the width and height of the bounding box enclosing the source pin and load pin $j$ of net $n_{i}$, respectively.

$c_{1}, c_{2} \quad$ : the capacitances of M1 and M2 per unit length, respectively.

$r_{1}, r_{2} \quad:$ the resistances of M1 and M2 per unit length, respectively.

$R_{i 0} \quad:$ the equivalent output resistance of the source.

$\sum_{k} C_{l k} \quad$ : the sum of the load capacitances.

In the following, we abbreviate $\operatorname{del}_{i j}\left(w_{i}, h_{i}, l 1_{i j}, l 2_{i j}\right)$ to $d e l_{i j}$ for simplicity.

In general, a timing constraint can be specified between a primary input or an output of flip-flops(FFs) and a primary output or inputs of FFs. However, soft blocks are not pre-designed in the hierarchical design of the building block layout and hence the timing constraints are separately treated as the timing constraints of the inside and outside of soft blocks. Therefore, in this paper, we assume that a timing constraint is given between the source pin and a load pin of net $n_{i}$. Thus we specify a timing constraint of the source pin and a load pin $j$ of net $n_{i}$ as the maximum allowable propagation delay $D l_{a l l o w_{i j}}$ from the source to the load. Consequently, if $d e l_{i j} \leq D_{e l_{a l l o w}}$, then the timing constraint is satisfied.

Now, we formulate the timing-driven floorplanning problem (TDFP) as follows. Given (1) a set of blocks $\mathcal{M}=\mathcal{M} h \cup \mathcal{M} s$, (2) a set of nets $\mathcal{N}$, (3) timing constraints $\mathcal{T}$, and (4) physical parameters as input, the problem is to determine a legal floorplan of $\mathcal{M}$ which minimizes the chip area under the conditions; (1) no two blocks overlap, (2) $\forall m s_{i} \in \mathcal{M} s, r_{l}\left(m s_{i}\right) \leq r\left(m s_{i}\right) \leq$ $r_{u}\left(m s_{i}\right),(3) \forall m s_{i} \in \mathcal{M} s, a\left(m s_{i}\right)=w\left(m s_{i}\right) \times h\left(m s_{i}\right)$, and (4) satisfy the timing constraints $\mathcal{T}$.

\section{A TIMING-DRIVEN FLOORPLANNING ALGORITHM}

The proposed algorithm consists of two phases: (1) construction of a timingdriven topological arrangement with nonlinear programming, (2) conversion of the topological arrangement into a legal floorplan. In the following, we present the details of each phase.

\subsection{Timing-Driven Topological Arrangement with Nonlinear Programming (Phase 1)}

The objective of phase 1 is to obtain a topological arrangement of blocks so as to minimize the total wire length under the given timing constraints. To achieve the objectives, we transform the topological arrangement problem to a mathematical programming problem. But mathematical programming tends to require much computation time and memory space. Therefore we 
apply mathematical programming to subcircuits, for which the formulated problem can be solved in a practical computation time and with practical size of memory space.

In order to obtain an initial topological arrangement, we apply the timingdriven placement algorithm for standard cell layout we have presented in (Koide et al. 1995). However, the width and height of blocks are much larger than those of standard cells and some blocks may be overlapped each other. To minimize overlaps between blocks, we select a target subcircuit and add a set of constraints that specify a minimum separation of each pair of blocks. Then we formulate the problem into a nonlinear programming problem with the timing and minimum separation constraints, and obtain a new topological arrangement by solving it. Those operations are repeated until the topological arrangement satisfies the timing constraints and the ratio of overlaps among blocks becomes less than a pre-determined value. In the following, first, we explain selection of a target subcircuit and then formulate the topological arrangement problem as a nonlinear programming problem.

\section{(a) Selection of a Target Subcircuit}

Now, we define a target subcircuit as $\mathcal{L}_{\text {mov }}=\left(\mathcal{M}_{\text {mov }}, \mathcal{N}_{\text {mov }}\right)$, where $\mathcal{M}_{\text {mov }}$ is a set of blocks, called movable blocks, of the subcircuit, and $\mathcal{N}_{\text {mov }}$ is a set of nets, called movable nets, each connecting to at least one movable blocks. Cells other than movable blocks in the whole circuit are called fixed blocks and their set is represented by $\mathcal{M}_{\text {fix }}\left(=\mathcal{M}-\mathcal{M}_{\text {mov }}\right)$. Nets other than movable nets in the whole circuit are called fixed nets and their set is represented by $\mathcal{N}_{\text {fix }}\left(=\mathcal{N}-\mathcal{N}_{\text {mov }}\right)$.

First, we find one of nets with a large violation ratio. The violation ratio is the value of actual delay time of a net divided by the allowable delay time of it, i.e., $d l_{i j} / D_{e l_{a l l o w i j}}$. The candidate nets are selected from the largest $10 \sim 20$ percent in all the nets in point of the violation ratio. But, to improve the topological arrangement in a small number of iteration, we don't select any nets which have been selected in the last $k(>0)$ iterations. Next, let a set of blocks which are connected to the selected net be the initial subcircuit. Then the subcircuit is expanded by adding blocks one by one. The added block should have large connectivity, which is the number of connections to the present subcircuit. To avoid repeatedly selecting the same block to be added to the target subcircuit at each iteration, we introduce a randomness in the selection step and determine whether the block is included. If included, all the nets connecting to it are redefined as a set of movable nets.

To solve the problem in a practical computation time, we must limit the number of variables of the mathematical programming. Hence, the growing process of the subcircuit continues until the number of variables of the mathematical programming problem reaches to a given constant.

\section{(b) NLP Formulation of the Problem}

Now, we show how to formulate the placement problem into a nonlinear programming(NLP) problem. We define variables to represent the wire lengths of 
movable nets as well as the positions of movable blocks. Let $x_{j}$ and $y_{j}$ be the center $X$ and $Y$ coordinates of movable block $m_{j} \in \mathcal{M}_{\text {mov }}$, respectively. Denote by $w_{i}$ and $h_{i}$ the width and height of the bounding box of net $n_{i} \in \mathcal{N}_{\text {mov }}$, respectively. Let $\mathcal{M}_{j}$ be a set of blocks connecting to net $n_{j}$. Then, we can represent the bounding box of movable net $n_{i}$ by the following inequalities:

$$
\left.C N 1: \begin{array}{l}
x_{j}-x_{k} \leq w_{i} \\
y_{j}-y_{k} \leq h_{i}
\end{array}\right\} \begin{aligned}
& \forall m_{j} \neq m_{k} \in \mathcal{M}_{i} \cap \mathcal{M}_{\text {mov }}, \\
& \forall n_{i} \in \mathcal{N}_{\text {mov }}
\end{aligned}
$$

These inequalities mean that for any pair of movable blocks connecting to $n_{i}$, they are completely included in the bounding box of $n_{i}$. When $n_{i}$ is connected to fixed blocks, the following inequalities should be satisfied:

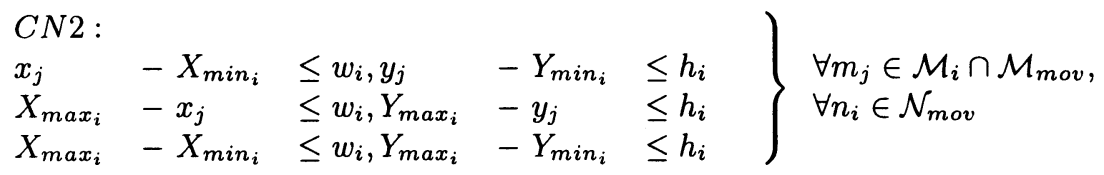

where $X_{\min _{i}}$ and $X_{\max _{i}}$ (resp. $Y_{\min _{i}}$ and $Y_{\max _{i}}$ ) are the minimum and maximum X (resp. Y) coordinates in fixed blocks of $\mathcal{M}_{i}$. If a net has some fixed blocks, the bounding box of the fixed blocks can be constructed, and for any pair of a movable block and the bounding box, their bounding box is completely included in the bounding box of $n_{i}$.

To calculate delay $d e l_{i j}$ from the source to load $j$ of net $n_{i}$, we introduce variables $l 1_{i j}, l 2_{i j}$ to represent the horizontal and vertical lengths of the bounding box of the source and load $j$.

$$
\left.C S: \begin{array}{l}
x_{k}-x_{l} \leq l 1_{i j}, y_{k}-y_{l} \leq l 2_{i j} \\
x_{l}-x_{k} \leq l 1_{i j}, y_{l}-y_{k} \leq l 2_{i j}
\end{array}\right\} \forall n_{i} \in \mathcal{N}_{\text {mov }}
$$

where $\left(x_{k}, y_{k}\right)$ and $\left(x_{l}, y_{l}\right)$ denote the center coordinates of cells corresponding to the source and sinks of $n_{i}$.

As mentioned in Section 2, the timing constraints are specified by pairs of the source pin and a load pin $j$ of net $n_{i} \in \mathcal{N}$. Then the constraints of net delay can be written as follows.

$C D: \quad \operatorname{del}_{i j}\left(w_{i}, h_{i}, l 1_{i j}, l 2_{i j}\right) \leq$ Del $_{\text {allow }}, \quad \forall n_{i} \in \mathcal{N}_{\text {mov }}$

To control block overlaps during phase 1 , we specify a minimum separation between two blocks. The minimum separation is expressed by the Manhattan distance between the centers of two blocks. The distance between blocks $m_{j}$ and $m_{k}$, denoted $d i s t_{j k}$, is defined as, dist $j_{j k}=\sqrt{\left(x_{j}-x_{k}\right)^{2}+\left(y_{j}-y_{k}\right)^{2}}$. In phase 1 of the algorithm, we do not have to delete all the overlaps because phase 2 does. To avoid over-constraining the problem, we set the minimum separation between $m_{j}$ and $m_{k}$ to

$\operatorname{sep}_{j k}=\eta_{j k} \times \max \left\{\frac{w\left(m_{j}\right)+w\left(m_{k}\right)}{2}, \frac{h\left(m_{j}\right)+h\left(m_{k}\right)}{2}\right\}$,

where $w\left(m_{j}\right)$ and $h\left(m_{j}\right)$ are the width and height of $m_{j}$, respectively, and $\eta_{j k}$ denotes a parameter $\left(0 \leq \eta_{j k} \leq 1\right)$. We initially set $\eta_{j k}$ to 0.5 and dynami- 
cally increase the value up to 1 during the iterative improvement step. If we consider the minimum separation constraints for any pair of blocks, we need $M(M-1) / 2$ constraints where $M$ is the number of blocks. To solve the mathematical programming problem in a practical computation time, we therefore select the target subcircuit $\mathcal{L}_{\text {mov }}=\left(\mathcal{M}_{\text {mov }}, \mathcal{N}_{\text {mov }}\right)$ and apply mathematical programming to $\mathcal{L}_{\text {mov }}$. Then the constraints of minimum separation can be written as follows.

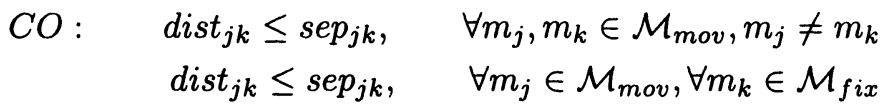

Now, we formulate the topological arrangement problems without/with the minimum separation constraints as the following nonlinear programming problems.

[NLP without/with the minimum separation constraints]

$\begin{array}{rll}\text { minimize }: & \sum \sigma_{i} \in \mathcal{N}_{\text {mov }}\left(w_{i}^{2}+h_{i}^{2}\right) \\ \text { subject to }: & C N 1 \cup C N 2 \cup C S \cup C D \\ \text { subject to }: & C N 1 \cup C N 2 \cup C S \cup C D \cup C O\end{array}$

where $\sigma_{i}$ denotes a constant considering the criticality of net $n_{i}$.

\section{(c) The Algorithm of Phase 1}

The proposed algorithm iteratively improves a topological arrangement so as to minimize the wire length and to satisfy the timing constrains and the minimum separation constraints. First, the algorithm selects all the blocks as the target subcircuit and formulates the NLP without the minimum separation constraints (8) for the target subcircuit. Then an initial topological arrangement is generated by solving the NLP problem. At each iteration, the algorithm performs timing verification and calculates timing violation ratios for all the nets. Then, it constructs a target subcircuit, solves the formulated nonlinear programming(NLP) problem with the separation constraints (9), and places blocks in the target subcircuit based on the result of NLP problem. These processes are repeated until the maximum violation ratio and the overlapping ratio become less than pre-determined values or the iteration count reaches a preset value. In the current implementation, we use the MINOS 5.4 (Murtagh \& Saunders 1995) package to solve the NLP problem.

\subsection{Floorplanning with Topological Constraint Manipulation ( Phase 2)}

In phase 2, we determine the block attributes (e.g., absolute locations, dimensions of soft blocks) so as to minimize the chip area. The output from phase 1 is a topological arrangement optimized for timing and connectivity. In order to get a final legal floorplan, we must remove all overlap among blocks. 


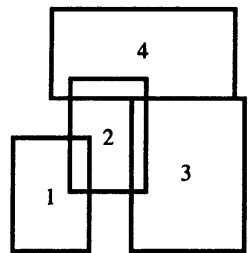

(a) phase 1
$\mathrm{G}_{\mathbf{H}}$

$\mathrm{G}_{\mathrm{V}}$

(b) topological constraints

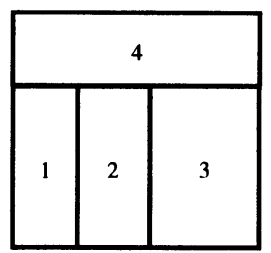

(c) phase 2

Figure 2 A topological arrangement of 4 blocks and the corresponding topological constraint set.

This phase is performed without undoing any of the decisions of the first phase, i.e., topological proximity between blocks in the solution produced by the first phase is maintained. We adopt a constraint-based approach we have proposed in (Koide et al. 1994) to convert the topological arrangement into a legal floorplan.

The topological arrangement is interpreted as a set of topological constraints. A topological constraint set of blocks is given by two directed acyclic graphs $\left(G_{H}, G_{V}\right): G_{H}$ is a horizontal constraint graph and $G_{V}$ is a vertical constraint graph(Vijayan \& Tsay 1991). The node set of both $G_{H}$ and $G_{V}$ is exactly the set of blocks $\mathcal{M}$. If $\left(m_{i}, m_{j}\right), m_{i}, m_{j} \in \mathcal{M}$, is an edge in $G_{H}$, then $m_{i}$ is to be placed to the left of $m_{j}$. If $\left(m_{i}, m_{j}\right), m_{i}, m_{j} \in \mathcal{M}$, is an edge in $G_{V}$, then $m_{i}$ is to be placed below $m_{j}$. Figure 2 shows an example of the topological arrangement of blocks and the corresponding topological constraint set.

In phase 2, we consider the following floorplanning problem FTC (Floorplanning with Topological Constraint). Given a set of blocks, a strongly complete* constraint set $\left(G_{H}, G_{V}\right)$, and bounds of aspect ratio for each soft block, as input, the problem is to determine a legal floorplan, that is, the absolute locations of the blocks and dimensions of the soft blocks, so as to minimize the chip area under the constraints such that the soft blocks must satisfy the given bounds on aspect ratios and a floorplan must strongly respect the input topological constraint set.

The proposed algorithm in phase 2 is an extension of the algorithm presented in (Vijayan \& Tsay 1990, Vijayan \& Tsay 1991), which we refer to as the conventional algorithm, or the algorithm VT, in the following. The approach of the algorithm VT is to first derive a complete constraint set from the input relative placement and then to delete those redundant constraints on longest paths on $G_{V}$ and $G_{H}$ until no redundant edges in $G_{V}$ or $G_{H}$ exist. Next, soft blocks on longest paths on $G_{V}$ and $G_{H}$ are also reshaped for some constant times to further reduce the chip area. The algorithm repeats both the steps until no improvement of the solution is achieved.

${ }^{*} \mathrm{~A}$ constraint set $\left(G_{H}, G_{V}\right)$ is said to be strongly complete if for any pair of blocks $m_{i}$ and $m_{j}$, there is an edge connecting them in either $G_{H}$ or $G_{V}$ or both. 
First, we introduce some modification of the algorithm VT, which is used as a procedure in the proposed algorithm. Unlike the algorithm VT, we reshape all soft blocks on the longest path in both horizontal and vertical constraint graphs to reduce the chip area for some constant times. For soft blocks which are not on the selected longest path, we also reshape the soft blocks to make their aspect ratio close to one. In the following, the algorithm VT with the above modification is called the Modified Vijayan and Tsay algorithm (the algorithm MVT).

Next, we describe a tentative insertion(Koide et al. 1994) of a constraint. In the algorithm VT, the ordering of constraints to be deleted may influence the final results. To search in a wide range of solution space by changing the ordering of deletion of constraints, we may firstly modify the given constraint set by tentatively inserting a dummy constraint $s_{i}$, which is not contained in the original constraint set $\left(G_{H}, G_{V}\right)$. This constraint is called a soft constraint. On the other hand, a non-redundant constraint which is originally contained in the given constraint set $\left(G_{H}, G_{V}\right)$ is said to be a hard constraint. This tentative insertion of a soft constraint $s_{i}$ is said to be tentative insertion. In the proposed algorithm, we tentatively insert a soft constraint, and check whether one of the longest paths on the constraint graphs $G_{H}$ and $G_{V}$ is changed. If it is, we actually insert the soft constraint, update the constraint set, and apply the algorithm MVT. To improve the obtained solution, we also reinsert not one deleted constraint but all deleted constraints $\left(m_{j}, m_{i}\right)$ relative to each block $m_{i} \in \mathcal{M}$ in the previous step and apply MVT again to obtain a final result.

During the algorithm execution, the algorithm keeps the tentatively best solution. When updating the tentatively best solution, the algorithm checks not only the chip area but also timing violation. Thus, the final solution of phase 2 is assured to satisfy the timing constraint.

\section{EXPERIMENTAL RESULTS}

The proposed algorithm has been implemented in $\mathrm{C}$ on a UltraSPARC workstation, and tested for MCNC benchmark data. In the experiments, we used "ami33" (123 nets, 33 blocks, 42 I/O pins, and 92 timing constraints) and generated two data "ami33a" and "ami33b" by changing positions of I/O pads in "ami33". In the experiments, all blocks are regarded as soft blocks. Since there is no timing information of the original circuits, we hence estimated the delay of each net based on a floorplanning result which was obtained by a non-timing-driven floorplanning algorithm, and gave a timing constraint for each net multiplying the estimated delay by $0.9 \sim 1.2$.

To show the effectiveness of phase 1 of the proposed floorplanning algorithm, we compared the results of phase 1 of the proposed timing-driven algorithm with the proposed algorithm without considering the timing constraints. In the experiments, we set the permissible violation ratio, the overlapping ratio 
Table 1 Experimental results of phase 1 of the floorplanning.

\begin{tabular}{ccccccc}
\hline data & Algo & Length & \#vio & Max vio & Overlap ratio & Time(sec) \\
\hline \multirow{2}{*}{ ami33 } & without & 73518 & 8 & 1.24 & 0.19 & 3145.5 \\
& with & 73432 & 0 & 0.98 & 0.17 & 3136.3 \\
\hline \multirow{2}{*}{ ami33a } & without & 95648 & 9 & 1.21 & 0.17 & 3885.0 \\
& with & 96479 & 0 & 0.98 & 0.12 & 3082.0 \\
\hline \multirow{2}{*}{ ami33b } & without & 94638 & 8 & 1.47 & 0.15 & 3715.3 \\
& with & 96426 & 0 & 0.96 & 0.19 & 4769.0 \\
\hline
\end{tabular}

Table 2 Experimental results of phase 2 of the floorplanning algorithm.

\begin{tabular}{ccccccc}
\hline data & Algo & Area & Length & \#vio & Max vio & Time(sec) \\
\hline \multirow{2}{*}{ ami33 } & conv & 1507695 & 72379 & 2 & 1.30 & 5.0 \\
& pro & 1562697 & 78032 & 0 & 0.87 & 114.8 \\
\hline \multirow{2}{*}{ ami33a } & conv & 1873632 & 100247 & 7 & 1.50 & 3.4 \\
& pro & 1706821 & 94501 & 0 & 0.98 & 156.2 \\
\hline \multirow{2}{*}{ ami33b } & conv & 1526887 & 96858 & 7 & 1.53 & 3.6 \\
& pro & 1479049 & 99328 & 0 & 0.98 & 108.0 \\
\hline
\end{tabular}

of blocks, and the maximum iteration count to $0.80,0.2$, and 300 , respectively. Table 1 shows the results of phase 1 . In the table, "without" and "with" mean the proposed algorithms without and with considering the timing constraints, respectively. "Length" is the estimated total wire length, that is, the sum of half the perimeter length of the bounding box for each net. The term "\#vio" is the number of violated timing constraints and "Max vio" is the maximum violation ratio for the given timing constraints. "Overlap ratio" is the ratio of the sum of the overlapped area among all the blocks to the total area of blocks.

From the results in Table 1, while the algorithm without considering the timing constraints violates some timing constraints, the proposed algorithm can produce the comparable results without any timing violations. As for the running time, since the proposed algorithm solves the NLP problem which takes the minimum separation constraints as well as the timing constraints into consideration, the running time of the algorithm takes about one hour. However, the number of blocks in the building block layout is generally small and therefore we think this running time is still considered practical.

Next, in Table 2, we show the floorplanning results obtained by applying the detailed placement step (phase 2) to the results of phase 1 in Table 1. For the results of phase 1 of the proposed timing-driven algorithm, we performed phase 2 of the proposed algorithm presented in Section 3.2. On the other hand, for the results of phase 1 of the algorithm without timing constraints, we ap- 
plied the algorithm VT(Vijayan \& Tsay 1991). In Table 2, "pro" means the proposed floorplanning algorithm and "conv" denotes the conventional algorithm which does not consider the timing constraints in the global placement step and applies the algorithm VT in the detailed placement step. "Area" denotes the area of the minimum rectangle enclosing all the blocks. From the results, the proposed floorplanning algorithm can successfully produce results without any timing violations within a practical computation time. Since phase 1 of the proposed algorithm can produce the global placements that satisfy the given timing constrains and have few overlapped area, phase 2 of the proposed algorithm can produce the floorplanning without timing violations by maintaining topological proximity between blocks in the solution produced by phase 1 .

\section{CONCLUSION}

In this paper, we proposed a timing-driven floorplanning algorithm based on nonlinear programming and topological constraint manipulation. The proposed algorithm consists of two phases. The topological arrangement in the first phase is iteratively improved by solving the nonlinear programming problem for a target subcircuit. The second phase transforms this topological arrangement into a legal floorplan and determines the positions of blocks and shapes of soft blocks so as to minimize the chip area under the given timing constraints. Through the simulation experiments, the proposed algorithm can produce floorplans without any timing violations in a practical computation time. Future research includes a reduction of the computation time of the algorithm and an extension of the algorithm to optimize the dimensions of blocks in the first phase.

\section{REFERENCES}

Bakoglu, H. B. (1990), Circuits, Interconnections, and Packaging for VLSI, AddisonWesley Publishing Company, Inc.

Elmore, W. C. (1948), 'The transient response of damped linear networks with particular regard to wideband amplifiers', J. Appl. Phys. 19, 55-63.

Gao, T., Vaidya, P. M. \& Liu, C. L. (1992), A performance driven macro-cell placement algorithm, in 'Proc. 29th Design Automation Conference', pp. 153-158.

Koide, T., Katsura, Y., Wakabayashi, S. \& Yoshida, N. (1994), A floorplanning method with topological constraint manipulation, in 'Proc. of 1994 IEEE International Symposium on Circuits and Systems', Vol. 1, pp. 165-168.

Koide, T., Ono, M., Wakabayashi, S., Nishimaru, Y. \& Yoshida, N. (1995), A new performance driven placement method with the Elmore delay model for row based VLSIs, in 'Proc. of Asia and South Pacific Design Automation Conference', pp. 405-412.

Kuh, E. S. \& Shih, M. (1992), Recent advances in timing-driven physical design, in 'Proc. Asia-Pacific Conference on Circuits and Systems', pp. 23-28. 
Lengauer, T. (1990), Combinatorial Algorithms for Integrated Circuit Layout, John Wiley \& Sons.

Murtagh, B. A. \& Saunders, M. A. (1995), MINOS 5.4 user's guide, Technical Report SOL 83-20R, Department of Operations Research, Stanford Univ.

Ogawa, Y., Pedram, M. \& Kuh, E. S. (1990), Timing-driven placement for general cell layout, in 'Proc. International Symposium on Circuits and Systems', pp. 872-875.

Prasitjutrakul, S. \& Kubitz, W. J. (1989), Path-delay constrained floorplanning: A mathematical programming approach for initial placement, in 'Proc. Design Automation Conference', pp. 364-369.

Sait, S. M., Youssef, H., Tanvir, S. \& Benten, M. S. T. (1994), Timing influenced general-cell genetic floorplanner, in 'Proc. European Design Automation Conference'.

Tia, T.-. S. \& Liu, C. L. (1993), A new performance driven macro-cell placement algorithm, in 'Proc. European Design Automation Conference', pp. 66-71.

Vijayan, G. \& Tsay, R.-S. (1990), Floorplanning by topological constraint reduction, in 'Proc. International Conference on Computer-Aided Design', pp. 106-109.

Vijayan, G. \& Tsay, R.-S. (1991), 'A new method for floorplanning using topological constraint reduction', IEEE Trans. on Comput. -Aided Des. Integrated Circuits \& Syst. CAD-12(10), 1494-1501.

Youssef, H., Sait, S. M. \& Al-Farra, K. J. (1995), Timing influenced force directed floorplanning, in 'Proc. European Design Automation Conference', pp. 156161.

\section{BIOGRAPHY}

Tetsushi Koide was received the B.E. degree in Physical Electronics and the M.E. degree in Systems Engineering from Hiroshima University in 1990 and 1992, respectively. He is currently a Research Associate in Faculty of Engineering at Hiroshima University. His research interests include combinatorial optimization and VLSI CAD. Mr. Koide is a member of the Association for Computing Machinery, the Institute of Electrical and Electronics Engineers, the Institute of Electronics, Information and Communication Engineers of Japan, and the Information Processing Society of Japan.

Shin'ichi Wakabayashi was received the B.E. degree in Electrical Engineering, M.E. and Ph.D. degrees in Systems Engineering from Hiroshima University in 1979, 1981, and 1984, respectively. He was a researcher at Tokyo Research Laboratory, IBM Japan Ltd., in 1984-1988. Since 1988 he has been an Associate Professor in Faculty of Engineering, Hiroshima University. His research interests include VLSI $\mathrm{CAD}$, combinatorial optimization, parallel and distributed algorithms, and theory of computing. Dr. Wakabayashi is a member of the Association for Computing Machinery, the Institute of Electrical and Electronics Engineers, the Institute of Electronics, Information and Communication Engineers of Japan, and the Information Processing Society of Japan. 